\title{
BMJ Open Energy conservation education intervention for people with end-stage kidney disease receiving haemodialysis (EVEREST): protocol for a cluster randomised control trial
}

\author{
Sita Sharma (1) , ${ }^{1,2}$ Kimberly E Alexander, ${ }^{3}$ Theresa Green, ${ }^{4,5}$ Min-Lin (Winnie) Wu, ${ }^{1,2}$ \\ Ann Bonner (1) 1,2,6
}

To cite: Sharma S, Alexander KE, Green T, et al. Energy conservation education intervention for people with end-stage kidney disease receiving haemodialysis (EVEREST): protocol for a cluster randomised control trial. BMJ Open 2022;12:e056544. doi:10.1136/ bmjopen-2021-056544

- Prepublication history for this paper is available online. To view these files, please visit the journal online (http://dx.doi. org/10.1136/bmjopen-2021056544).

Received 18 August 2021 Accepted 28 January 2022

Check for updates

(c) Author(s) (or their employer(s)) 2022. Re-use permitted under CC BY-NC. No commercial re-use. See rights and permissions. Published by BMJ.

For numbered affiliations see end of article.

Correspondence to

Sita Sharma;

sita.sharma@griffithuni.edu.au

\section{ABSTRACT}

Introduction Multiple symptoms occur in people with kidney failure receiving haemodialysis (HD) and these symptoms have a negative impact on health-related quality of life (HRQoL). Fatigue, the most common symptom, is debilitating and difficult to manage. Educational interventions involving energy conservation strategies are helpful in reducing fatigue, however the effectiveness of energy conservation has not been previously studied in those receiving HD. The aim of this study is to evaluate the effectiveness of an energy conservation education intervention for people with endstage kidney disease receiving HD (EVEREST trial). Methods and analysis A pragmatic cluster randomised control trial with repeated measure will be used. One hundred and twenty-six participants from tertiary level dialysis centre will be cluster randomised to the intervention and control group according to HD treatment day. The intervention group will receive usual care along with a structured energy conservation education programme over 12 weeks comprising three individual face-to-face educational intervention sessions, one booster session and a booklet. The control group will receive usual care from their healthcare providers and a booklet at the end of the study. The primary outcome is fatigue, and the secondary outcomes are other Chronic Kidney Disease (CKD) symptoms, occupational performance and HRQoL. Intention-to-treat analysis will occur and will include a change in primary and secondary outcomes.

Ethics and dissemination Ethical approval has been obtained from the Human Research Committee of the Griffith University and Nepal Health Research Council. The results of this research will be published and presented in a variety of forums.

Trial registration number NCT04360408.

\section{INTRODUCTION}

Chronic kidney disease (CKD) is both a major global public health problem and contributor to the overall burden of non-communicable disease, ${ }^{1}$ affecting about $13 \%$ of the global population. $^{2}$ The internationally agreed
Strengths and limitations of this study

- This study used cluster randomisation according to the day of haemodialysis to prevent treatment contamination between groups.

- The intervention material was developed to be simple and easily understood by those with limited education.

- Participants are recruited from one haemodialysis centre in Nepal.

- This study is focused on those receiving haemodialysis limiting generalisability to this population.

definition of CKD is an estimated glomerular filtration rate $\left(\right.$ eGFR) $<60 \mathrm{~mL} / \mathrm{min} / 1.73 \mathrm{~m}^{2}$ for 3 months or longer. ${ }^{3}$ CKD is then classified into five grades based on eGFR. ${ }^{4}$ When eGFR $<15 \mathrm{~mL} / \mathrm{min} / 1.73 \mathrm{~m}^{2}$ then the term kidney failure (previously end-stage kidney disease) is used. ${ }^{4}$ While there is no kidney registry in Nepal, the prevalence of kidney failure is approximately $2900 .^{5}$ Haemodialysis (HD) is the most common modality of treatment for those with kidney failure and usually prescribed three times per week, with a duration of 4-5 hours per session. ${ }^{6}$ It can be performed either in-centre in a hospital or a satellite unit or at home. ${ }^{7}$ This treatment impacts on almost all aspects of daily life leading to decreased health-related quality of life (HRQoL) ${ }^{8}$ including maintaining employment and being able to undertake routine social activities ${ }^{9}$ as well as affecting family members. ${ }^{10} 11$

Physical and psychological symptoms are common in this population and may be related to the underlying pathologies, presence of multiple comorbidities, accumulation of uraemic toxins or fluids, medication side effects and inadequacy of dialysis. ${ }^{12}$ 
Several studies have described symptom burden in this population. ${ }^{12-16}$ Almutary et al, who compared those not on dialysis with those receiving either HD or peritoneal dialysis, reported that both symptom prevalence and severity was highest in those receiving HD, and that about $85 \%$ reported being fatigued. ${ }^{13}$

Fatigue is an overwhelming subjective experience of discomfort associated with physical and mental exhaustion. ${ }^{17}$ Fatigue in people with kidney failure negatively impacts individuals' day-to-day activities, ${ }^{18}{ }^{19}$ HRQoL, ${ }^{20}$ increases hospitalisations and mortality. ${ }^{21}$ Various factors have been associated with fatigue in kidney failure such as demographic characteristics, ${ }^{22}$ elevated urea levels, anaemia, depression, anxiety and sleep disturbances. ${ }^{22} 23$ Medication side effects and HD treatment-related factors like dialysis inadequacy and excessive ultrafiltration have been associated with fatigue. ${ }^{24-26}$

Managing fatigue is essential in improving HRQoL for individuals on HD. It can be argued that adults receiving HD can benefit from symptom self-management techniques to reduce fatigue and other CKD symptoms. ${ }^{27}$ Previous research has shown that exercise could reduce fatigue ${ }^{2228}$ however barriers such as having sufficient available expert staff, dialysis-related fatigue, comorbid conditions and lack of motivation may limit the ability for some to be actively involved in exercise. ${ }^{29}$ Educational interventions are believed to improve cancer-related fatigue ${ }^{30}$ and there is some evidence to support this in earlier stages of CKD. ${ }^{31}$ Likewise, education about energy conservation is another approach to manage fatigue that has shown a significant reduction in fatigue in other chronic diseases, including multiple sclerosis ${ }^{32}$ and cancer. ${ }^{334}$ However, its effectiveness is yet to be tested in the HD population.

In a systematic review, Blikman et a $\hat{P}^{2}$ included six interventional studies which examined the effects of energy conservation management (ECM) for fatigue and HRQoL in people with multiple sclerosis (MS). Four studies included in this review used an ECM intervention programme based on Packers' 'Managing Fatigue' course and two were guided by the MS fatigue guidelines. Interventions in these studies were delivered in group format and face-to-face except one study ${ }^{35}$ where the intervention was delivered via teleconference. Meta-analysis of two studies ${ }^{35} 36$ included in this review showed that an ECM intervention was more effective than no intervention in reducing the impact of fatigue measured by fatigue impact scale (FIS). ${ }^{32}$ There was an improvement in the FIS subscale significantly on the cognitive subscale (Mean Difference (MD) $=-2.91 ; 95 \%$ CI -4.32 to -1.50 ), the physical subscale $(\mathrm{MD}=-2.99 ; 95 \% \mathrm{CI}-4.47$ to -1.52$)$ and the psychological subscale $(\mathrm{MD}=-6.05 ; 95 \% \mathrm{CI}-8.72$ to -3.37$){ }^{32}$ The same study also revealed that an ECM improved three domains of HRQoL, namely role physical (MD=17.26; 95\% CI 9.69 to 24.84), social functioning $(\mathrm{MD}=6.91 ; 95 \% \mathrm{CI} 1.32$ to 12.49$)$ and mental health ( $\mathrm{MD}=5.55 ; 95 \%$ CI 2.27 to 8.83 ). Another study evaluated the effect of energy conservation strategies in persons with breast cancer experiencing fatigue. ${ }^{33}$ In this study, the intervention was delivered face-to-face in the form of small group discussion. Duration of each session was $90 \mathrm{~min}$ and sessions were conducted weekly for 5 weeks. ${ }^{33}$ The result of this study showed that energy conservation strategies significantly reduced cancer-related fatigue in persons with breast cancer over an 8-week follow-up period $(F=69.8, \mathrm{p}<0.001){ }^{33}$

Despite fatigue being highly prevalent in those receiving HD, a recent systematic review did not find any interventional studies that used an educational approach for selfmanagement to reduce symptoms and improve HRQoL in people undergoing HD. ${ }^{37}$ Thus, this study aims to evaluate the effectiveness of an energy conservation education intervention for people with end-stage kidney disease receiving haemodialysis (EVEREST trial) in Nepal.

\section{Research hypotheses}

People with kidney failure on HD who receive energy conservation education (ECE) programme and usual care are more likely to have:

H1: reduced fatigue severity, frequency and perceived interference compared with people undergoing HD who received only usual care.

H2: reduced other CKD symptoms score compared with people undergoing HD who received only usual care.

H3: improved occupational performance and satisfaction compared with people undergoing HD who received only usual care.

H4: improved HRQoL compared with people undergoing HD who received only usual care.

\section{METHODS AND ANALYSIS \\ Study design}

A pragmatic cluster randomised control trial design with outcome assessor blinded, with 1:1 randomisation will be used. A pragmatic design attempts to find an answer to whether an intervention will work under usual conditions in a real clinical setting where few exclusion criteria are applied. ${ }^{38}$ To avoid possible treatment contamination, because participants on the same HD day may interact with each other as they spend approximately 4 hours in close proximity to each other during dialysis, a cluster design is used with cluster cohorts based on the day of dialysis. The Consolidated Standards of Reporting Trials flow diagram in figure 1 presents the study design.

\section{Study setting}

The study will be conducted at the National Kidney Centre (NKC), Kathmandu, Nepal. The NKC is the nonprofit, non-governmental organisation with the largest HD treatment facility in Nepal. The NKC provides HD treatment for around 240 people a month. Haemodialysis is free to all patients as mandated by the Ministry of Health and Population, Nepal, although medications and routine blood tests require patients to fund.

\section{Sample size}

G power software was used to calculate the sample size by performing the priori power analysis for an independent 


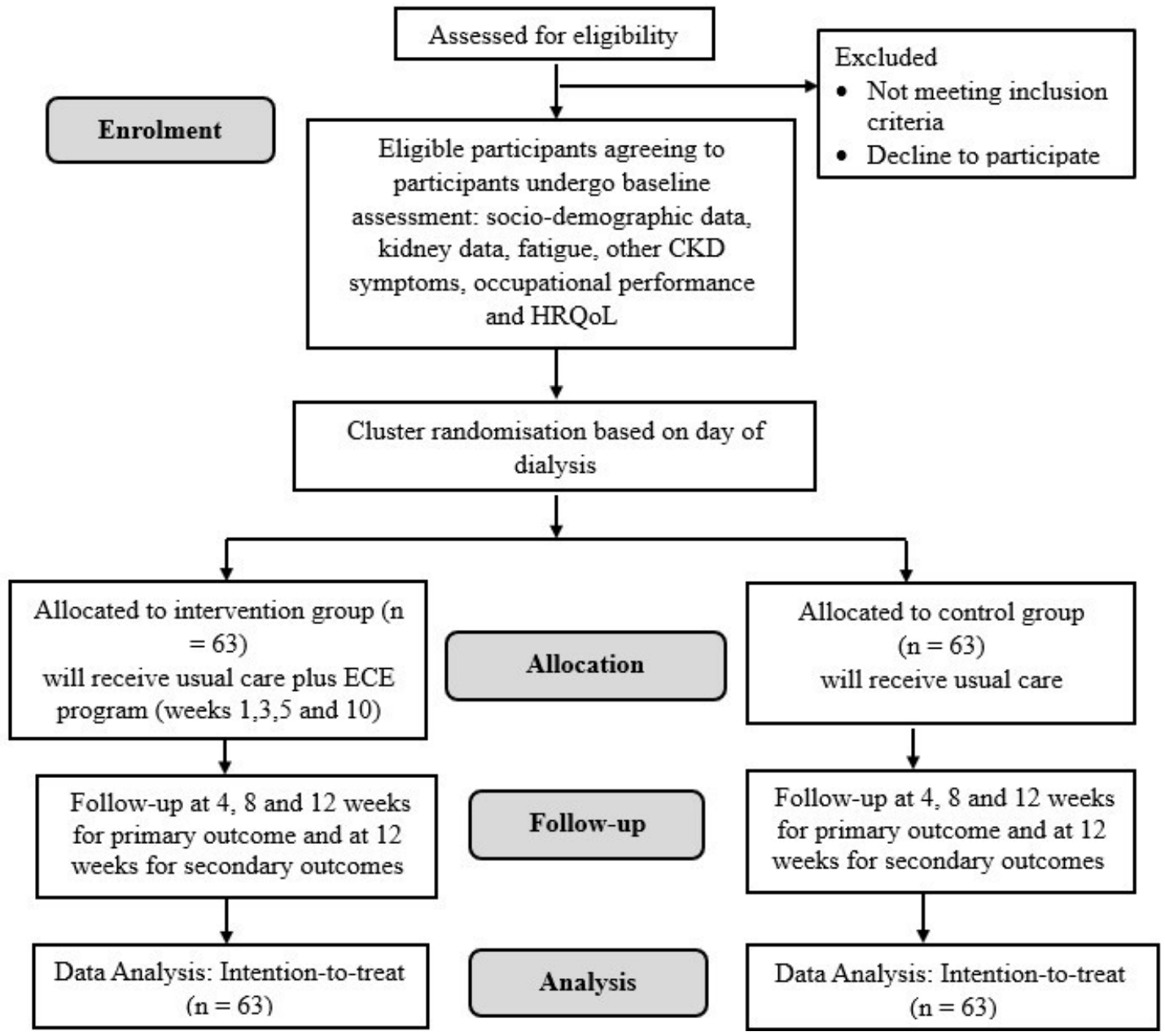

Figure 1 Consolidated Standards of Reporting Trials flow diagram: extension to cluster, reflecting the flow of participants in the study. CKD, Chronic Kidney Disease; HRQoL, health-related quality of life.

group two-tailed t-test. The sample size was calculated based on a large effect size (Cohen's $d$ from 0.90 to 1.5) taken from a previous study in patients with breast cancer on management of fatigue (measured with fatigue symptom inventory (FSI)) with yoga intervention. ${ }^{39}$ To have resultant $80 \%$ power, an effect size of 0.8 and a significance of $0.05,52$ participants are needed (26 in each group). As this study uses a cluster design, and assuming a moderate intra-cluster coefficient of 0.03 , the sample size is adjusted to compensate for design effect. To compensate for attrition and to avoid the possibility of non-normality of data, the calculation is further inflated by $20 \%$ and again $15 \%$, respectively. After adjusting for these, a final sample size of 126 (63 in each group) is required.

\section{Eligibility criteria}

Participants diagnosed with kidney failure and undergoing HD for $\geq 3$ months, aged 18 years and above, able to speak and understand Nepali language and willing to participate, will be included in this study. Participants who are in earlier grades of CKD or not dependent on HD, those acutely ill, diagnosed with cognitive impairment and those who are not willing to participate will be excluded. Participants may be withdrawn from the study at any time due to a safety concern, if they became sick or if unable to adhere with the trial procedure.

\section{Study intervention}

Intervention group

The intervention group will receive both usual care from their healthcare providers and the 12 weeks ECE programme. The ECE programme teaches individuals to recognise and modify their daily activities to reduce fatigue by analysing their daily work, home and leisure activities. ${ }^{40}$ This programme helps to develop a positive attitude towards decision-making and the maximum use of available energy ${ }^{32}$ It is designed to reduce the frequency, severity and impact of fatigue, increase a person's use of energy-conservation strategies and improve their confidence level and their ability to manage fatigue. ${ }^{32}$ Seven energy conservation strategies adopted from the 'Managing Fatigue' course developed by Packer et $a t^{41}$ form the content of the ECE programme (see table 1); each strategy is adapted to fit with the daily activities of Nepali people.

The ECE programme is guided by symptom management theory (SMT) as the theoretical framework. 
Table 1 Summary of the content of the energy conservation education programme

\begin{tabular}{|c|c|c|c|c|}
\hline Session & Goal and objectives & Topics & Duration & Teaching methods \\
\hline $\begin{array}{l}\text { Session } 1 \\
\text { (week 1) }\end{array}$ & $\begin{array}{l}\text { Goal: The goal of this session } \\
\text { is to provide information about } \\
\text { fatigue in kidney failure, its } \\
\text { causes and energy conservation } \\
\text { strategies and its application in } \\
\text { daily activities. } \\
\text { Objectives: } \\
\text { To set a friendly environment } \\
\text { and develop a trusting } \\
\text { interpersonal relationship. } \\
\text { To give information about } \\
\text { fatigue, its causes. } \\
\text { To give information about } \\
\text { energy conservation, energy } \\
\text { conservation strategy } 1 \\
\text { and its application in daily } \\
\text { activities. }\end{array}$ & $\begin{array}{l}\text { Fatigue in kidney failure. } \\
\text { Causes of fatigue. } \\
\text { Introduction of energy conservation. } \\
\text { Energy conservation strategies and its } \\
\text { application in daily activities. } \\
\text { - Strategy } 1 \text { : Organising daily routine } \\
\text { and evaluating priorities. } \\
\text { Summary. }\end{array}$ & $30-45 \mathrm{~min}$ & $\begin{array}{l}\text { A face-to-face session with the } \\
\text { help of recorded PowerPoint on } \\
\text { the laptop } \\
\text { question and answer } \\
\text { discussion. }\end{array}$ \\
\hline
\end{tabular}

(week 3)

\section{Session 2}

Goal: The goal of this session is to provide information about energy conservation strategies 2

3,4 and 5 , and their application in daily activities.

Objectives:

- To set a friendly environment and prepare participant for educational session.

- To revise the content of session 1.

- To provide opportunity to ask question about previous session.

- To give information about energy conservation strategies 2, 3, 4, 5 and examples relevant to daily activities.

$\begin{array}{ll}\text { Session 3 } & \text { Goal: The goal of this session } \\ \text { (week 5) } & \text { is to provide information about } \\ \text { energy conservation strategies } \\ 6 \text { and } 7 \text { and their application to } \\ \text { daily activities. } \\ \text { Objectives: } \\ \text { To set a friendly environment } \\ \text { and prepare participant for } \\ \text { educational session. } \\ \text { To revise session } 2 . \\ \text { To identify any concern about } \\ \text { previous session. } \\ \text { To provide opportunity to } \\ \text { ask question about previous } \\ \text { sessions. } \\ \text { To give information about } \\ \text { energy conservation } 6 \text { and } 7 \\ \text { and their application in daily } \\ \text { activities. } \\ \text { Goal: The goal of this session } \\ \text { is to revise the content of } \\ \text { all sessions with the help of } \\ \text { educational booklet. } \\ \text { Objectives: } \\ \text { To set a friendly environment } \\ \text { and prepare participant for } \\ \text { educational session. } \\ \text { bession } 4 \\ \text { (week 10) }\end{array}$

Revision of previous session.

Energy conservation strategies and its application in daily activities.

- Strategy 2: Simplifying the everyday task.

- Strategy 3: Organising station for activities and using the energyefficient appliances.

- Strategy 4: Pacing activities and avoid rushing.

- Strategy 5: The value of rest and Summary.
$30 \mathrm{~min}$

face-to-face session with the help of recorded PowerPoint on the laptop

question and answer discussion.
Revision of previous session.

Energy conservation strategies and its application in daily activities.

- Strategy 6: Communicating personal needs to others.

- Strategy 7: Using proper body

Summary
Revision of previous session.

Summarise the content of the booklet.

- Introduction of fatigue, energy conservation, its strategies and application in daily activities.
$30 \mathrm{~min}$

A face-to-face session with the help of recorded PowerPoint on the laptop question and answer discussion.
30-45 min A face-to-face session with the help of booklet question and answer discussion. 
This theory accounts for the person, health/illness, the environment, and includes the symptom experience, symptom management strategies and outcomes. ${ }^{42}$ Symptom management theory is built on the premise that a symptom experience is based on how an individual perceives, evaluates and responds to the symptom. ${ }^{43}$ Symptom management strategies used by individuals to delay a negative outcome of the symptom experience can be targeted by appropriate intervention strategies. The theory also explains that outcomes (eg, symptom status, functional status, emotional status, self-care, mortality, costs, HRQoL, and morbidity \& co-morbidity) may be altered by the symptom experience and/or symptom management strategies. The dynamic interaction between each dimension of SMT provides explicit and testable relationships among these dimensions. In this study, the relationship between a person's fatigue experience and other CKD symptoms, the ECE programme and outcomes including status of fatigue and other CKD symptoms, HRQoL and occupational performance will be tested using this theory.

\section{Face-to-face education session}

Four face-to-face educational sessions will be undertaken during participants' regular HD treatment. Sessions will be in weeks 1,3 , and 5 , followed by a booster session in week 10. Each session will be $30-45 \mathrm{~min}$ in duration. Research assistants (RAs; nurses trained by the principal researcher) will deliver the intervention to avoid information bias. Recorded PowerPoint presentations in simple Nepali language will be displayed on a laptop. At the end of each session, RAs will inform the participants of the date for the next session. This simple strategy will assist with participant retention in the study.

\section{Educational booklet}

Educational sessions will be supplemented by a booklet designed to be understood by an individual with minimal literacy to support a better understanding of fatigue in kidney failure, causes of fatigue, energy conservation strategies and their application in daily activities. Simple text information, along with informative images, will be used to assist participants to understand and apply the energy conservation strategies.

\section{Control group}

Participants randomised to the control group will receive usual care (standard care with no formalised, structured or tailored interventions to reduce symptom/s) from their healthcare providers. Participants in the control group will receive an ECE programme booklet at week 12 once the study is completed.

\section{Outcomes}

Primary outcome

Fatigue

The primary outcome of the study is fatigue, which is measured at Time $0=$ baseline, Time $1=$ week 4 , Time 2=week 8 and Time $3=$ week 12 using the FSI. ${ }^{44}$ This self-report instrument is comprised of 14 items assessing the frequency, severity, daily pattern of fatigue and its perceived interference with quality of life. Fatigue severity is measured by an 11-point item scale $(0=$ not at all fatigue, 10=as fatigued as I could be) that assesses least, average and most fatigue in the past week and right now. A composite fatigue score (FSI composite) will be derived by calculating the average across the three severity items. Frequency is measured as the number of days in the past week (0-7) that participants felt fatigue, as well as the percentage of each day on average they felt fatigued $(0 \%=$ none of the day; $100 \%=$ the entire day). Perceived interference, which assesses the degree to which fatigue in the past week was judged to interfere with the general level of activity, ability to bathe and dress, normal work activity, ability to concentrate, relations with others, enjoyment of life and mood, is measured on separate 11 -point scales $(0=$ no interference; $10=$ extreme interference). These interferences ratings can be summed (and averaged) to obtain a total perceived interference score. The final item provides qualitative information about possible diurnal variation in the daily experience of fatigue. The FSI has been used previously in a study on kidney failure population. ${ }^{45}$ This instrument was translated into the Nepali language, and has a Cronbach's alpha of $0.79 .^{46}$

\section{Secondary outcomes}

Secondary outcomes are other CKD symptoms, occupational performance and HRQoL, which will be measured at Time 0 (Baseline) and Time 3 (Week 12).

\section{Other CKD symptoms}

Other CKD symptoms will be measured using the Integrated Palliative Outcome Scale renal (IPOS-renal); designed to be used in CKD population including those receiving HD. This self-report instrument is short (11 items) making it quick and easy to administer. It measures the most common symptoms experienced by people with kidney disease; additional items such as information needs, practice issues; and anxiety of family. ${ }^{47}$ Question 2 of this instrument assesses 15 specific symptoms for each of these items, with responses rated 0 (no symptoms) to 4 (overwhelmingly). ${ }^{49}$ Questions 3-9 assess the psychological, spiritual, communication and practical problem or concern, for each of these, with responses also rated 0-4. Questions 1 and 11 are not scored. The overall IPOS-renal score can range from 0 to 92. The IPOS-renal demonstrates good reliability and validity ${ }^{50}$ however, it is not available in Nepali language. Prior to starting the study, the instrument was translated into Nepali. The process recommended by Sousa and Rojjanasrirat was used to translate the instrument in English to the Nepali language. ${ }^{51}$ A content validity index will be calculated while reliability of the instrument will be tested in a representative sample of HD participants in this study. 


\section{Occupational performance}

Occupational performance is measured by the Nepali version of the Canadian Occupational Performance Measure $(\mathrm{COPM}) .^{52}$ This instrument is designed to identify changes in occupational performance over a period of time. Administration of the COPM requires five steps. First, the individual identifies and prioritises everyday problems that restrict or impact the performance in the areas of self-care, productivity and leisure. Second, the identified problems are rated in terms of their importance on a scale of 1 (not important at all) to 10 (extremely important). Third, the five most urgent or important problems are identified on which to focus during the intervention. Fourth, the individual rates their performance and satisfaction. Both scales range from 1 to 10 , with higher values indicating better performance and greater satisfaction. After an appropriate interval (Week 12 in this study), performance and satisfaction are reassessed and calculated to measure changes overtime. Cronbach's alpha of this instrument for performance score was 0.89 , and for the satisfaction, the score was $0.88 .^{53}$

\section{Health-related quality of life}

Health-related quality of life is measured using the Nepali version of the SF-36 questionnaire. ${ }^{54}$ The SF-36 contains 36 multidimensional questions and has eight subscales: physical functioning, role physical, role emotional, energy/fatigue, emotional well-being, social functioning, pain and general health. ${ }^{55}$ There are two distinct concepts measured by the SF-36, represented by the physical component summary and mental component summary. ${ }^{55}$ For each subscale, items are scored using a Likert scale, summed and transformed on to a scale from 0 (worst health) to 100 (best health). ${ }^{55}$ This instrument has good reliability with Cronbach's alpha of $0.85 .^{54}$

\section{Additional measurements}

Demographic and clinical information, except blood results, will be collected at baseline only. The demographic tool has been designed to collect information about participants age, gender, residence, marital status, ethnicity, religion, type of family, educational status, occupation and income. Kidney characteristics such as CKD cause and duration, family history of CKD, duration and detail of HD treatment, past medical and surgical history, current medications will be extracted from hospital records. In addition, serum albumin, electrolytes and haemoglobin levels as well as iron studies results will be extracted from the individual's reports. These results will be collected at baseline then weeks 4, 8 and 12; aligned with assessment of fatigue. The intervention group will record levels of activity while at home prior to starting the ECE programme and then at week 12. Table 2 illustrates the timeline for measurement of outcomes and intervention sessions.

\section{Randomisation}

In this cluster randomised study, the unit of observation is at the level of the individual, and the unit of randomisation is at the level of dialysis day (cluster). Cluster randomisation will be done according to the $\mathrm{HD}$ day

\begin{tabular}{|c|c|c|c|c|c|c|c|c|c|c|c|c|}
\hline Time point (week) & 1 & 2 & 3 & 4 & 5 & 6 & 7 & 8 & 9 & 10 & 11 & 12 \\
\hline \multicolumn{13}{|l|}{ Enrolment } \\
\hline Informed consent & $x$ & & & & & & & & & & & \\
\hline Allocation & $x$ & & & & & & & & & & & \\
\hline Demographic information & $x$ & & & & & & & & & & & \\
\hline Clinical information & $x$ & & & & & & & & & & & \\
\hline Blood test results & $x$ & & & $x$ & & & & $x$ & & & & $x$ \\
\hline Fatigue & $x$ & & & $x$ & & & & $x$ & & & & $x$ \\
\hline Activity checklist & $x$ & & & & & & & & & & & $x$ \\
\hline \multicolumn{13}{|l|}{ Intervention } \\
\hline $\begin{array}{l}\text { Intervention: energy conservation education (ECE) } \\
\text { programme }\end{array}$ & ES1 & & ES2 & & ES3 & & & & & B & & \\
\hline Intervention: usual care & $x$ & $x$ & $x$ & $x$ & $x$ & $x$ & $x$ & $x$ & $x$ & $x$ & $x$ & $x$ \\
\hline Control: usual care & $x$ & $x$ & $x$ & $x$ & $x$ & $x$ & $x$ & $x$ & $x$ & $x$ & $x$ & $x$ \\
\hline
\end{tabular}

B, booster session; CKD, Chronic Kidney Disease; ES1, educational session 1; ES2, educational session 2; ES3, educational session 3. 
(Sunday/Tuesday/Thursday or Monday/Wednesday/ Friday) determined by an independent person, not directly involved in the study. This method of randomisation will avoid possible treatment contamination. Instances of participants attending different dialysis days and being potentially exposed to the intervention will be documented. An equal number of participants will be recruited from each cluster to ensure both intervention and control groups have an equal number.

\section{Recruitment and data collection}

The RAs will liaise with the dialysis nurse and medical doctor to identify eligible potential participants. A dialysis nurse who is taking care of the participant will seek approval from the participant to introduce the RA. Following confirmation, the RAs will approach potential participants to introduce herself, the purpose and methods of the study. The RAs will then assess all inclusion/exclusion criteria and invite them to participate. Potential participants will receive an information sheet and it will also be read out aloud (due to basic literacy concerns). Potential participants will also be given an opportunity to ask if there are any queries about the study before giving written consent. Baseline data will be collected by the RAs and entered into the Research Electronic Data Capture (REDCap) mobile application. Recruitment will occur until the required sample size is achieved. Comprehensive training is provided to RAs prior to starting the study.

\section{Blinding}

Due to the nature of the intervention and its pragmatic design, it will not be possible to blind participants, researcher, dialysis nurses or nephrologists. However, to minimise the risk of bias, a different RA, who will collect follow-up data, will be blinded to group allocation. Nephrologists and dialysis nurses who are responsible for caring for patients will not be involved in the allocation, delivery of the intervention or data collection. They may be aware of the allocation, but this will not affect the outcome of the study.

\section{Data management}

All data will be managed using the REDCap tool. It is a secure web application designed to support data capture for studies ${ }^{56}$ and it is suitable to use in Nepal. Hard copy material, including consent forms and activity checklist will be securely held in a locked filing cabinet. Electronic data will be stored in the secure research storage service managed by the university. The stored data will be accessible only by the research team. All identifiable information received from participants will be replaced with a unique code and stored separately. Data used in the analyses will remain in the de-identified format. No identifying information will be published. All data will be retained for at least 15 years after the end of the study, and then permanently deleted from the computer system or shredded.

\section{Data analysis}

First, a coding manual for each outcome variable will be developed with responses scored prior to entering the IBM SPSS statistics software V.28. Data will then be cleaned and checked to evaluate for missing data, any errors or invalid responses. Descriptive statistics will be used for all study variables. Baseline characteristics will be compared for the control and intervention group using independent t-test or Mann-Whitney $\mathrm{U}$ tests for continuous variables while $\chi^{2}$ or Fisher's exact tests for categorical variables. McNemar's test will be used to determine the difference in the activity before and after the intervention in the intervention group. Intention-to-treat principles using linear mixed models (LMM) will determine the differences between intervention and control groups between primary and secondary outcomes variables. This type of analysis is used to evaluate the effect of an intervention at different time points and also group-by-time interaction between groups. It is also possible to adjust the LMM due to the clustered nature of the data and include all randomised participants in the analysis. For continuous variables, when normality assumptions are not met, data will be transformed using log transformation. Effectiveness of intervention will be reported using mean differences with $95 \%$ CIs. Significance will be set at $p<0.05$.

\section{Trial status}

Trial is currently ongoing. Recruitment commenced in April 2021 and is expected to be completed in February 2022.

\section{Patient and public involvement}

There is no active involvement of patients or public in the development of this study protocol.

\section{ETHICS AND DISSEMINATION}

Ethical approval was obtained from the Human Research Committee of Griffith University and also the Ethical Review Board of Nepal Health Research Council prior to the commencement of the study. Appropriate approval was also sought from the research site. Informed written consent will be obtained from each participant before study commencement. Participants will be informed about voluntary participation and that there is no foreseeable risk or harm in participating in the study. Participants will be assured that their confidentiality will be maintained. Participants also have the liberty to discontinue participating in the study without any clarification. All personal data will be deidentified before analysis and reported collectively. The trial has been accepted by and registered in ClinicalTrials.gov on 23 April 2020.

The results of this research will be published and presented in a variety of forums. Manuscripts will be submitted to peer-reviewed journals and conference abstracts will be prepared for national and international conferences. 


\section{DISCUSSION}

There is growing evidence of a direct relationship between symptom burden and HRQoL in individuals with kidney failure ${ }^{57-59}$ however, the evidence to inform practice about improving HRQoL by targeting the most prevalent and severe symptom, fatigue, is not apparent. Energy conservation management does seem to improve the self-management of fatigue in other chronic diseases although its effectiveness for CKD-related fatigue is unknown. This study is needed as it will provide empirical evidence about the effectiveness of an ECE programme for fatigue management that can be integrated into the everyday life of people receiving HD.

Major limitations of this study are that recruitment of participants is from one dialysis centre in Nepal. This limitation is somewhat mitigated because the selected setting is one of the largest centres in the country referred for HD services. In addition, the study's population is limited to those receiving HD, which limits the generalisability of the finding to other CKD groups such as those receiving conservative treatment or other forms of kidney replacement therapy. This study will also use patient-reported outcome measures for symptoms, HRQoL and occupational performance; thus, it is difficult to identify whether participants will accurately report change in outcomes over time. Moreover, participants in this study will not be followed up for a long period after the intervention.

In conclusion, this study will evaluate the effectiveness of an ECE programme to reduce fatigue in people with kidney failure receiving HD. The evidence generated from this study is expected to positively influence patient outcomes by assessing symptoms and providing appropriate educational interventions during the HD session.

\section{Author affiliations}

${ }^{1}$ School of Nursing and Midwifery, Griffith University, Brisbane, Queensland, Australia

${ }^{2}$ Menzies Health Institute Queensland, Griffith University, Brisbane, Queensland, Australia

${ }^{3}$ School of Nursing, Queensland University of Technology, Brisbane, Queensland, Australia

${ }^{4}$ School of Nursing, Midwifery and Social Work, The University of Queensland, Brisbane, Queensland, Australia

${ }^{5}$ Surgical Treatment and Rehabilitation Service, Metro North Hospital and Health Service, Brisbane, Queensland, Australia

${ }^{6}$ Kidney Health Service, Royal Brisbane and Women's Hospital, Brisbane, Queensland, Australia

Contributors The study was conceived by SS, KEA, TG, M-LW and AB. SS led the literature review. All authors contributed to the preparation of the proposal, critical review and final approval of the manuscript.

Funding The authors have not declared a specific grant for this research from any funding agency in the public, commercial or not-for-profit sectors.

Competing interests None declared.

Patient and public involvement Patients and/or the public were not involved in the design, or conduct, or reporting, or dissemination plans of this research.

Patient consent for publication Not applicable.

Provenance and peer review Not commissioned; externally peer reviewed.
Open access This is an open access article distributed in accordance with the Creative Commons Attribution Non Commercial (CC BY-NC 4.0) license, which permits others to distribute, remix, adapt, build upon this work non-commercially, and license their derivative works on different terms, provided the original work is properly cited, appropriate credit is given, any changes made indicated, and the use is non-commercial. See: http://creativecommons.org/licenses/by-nc/4.0/.

\section{ORCID iDs}

Sita Sharma http://orcid.org/0000-0001-9136-1810

Ann Bonner http://orcid.org/0000-0001-9920-6743

\section{REFERENCES}

1 Jha V. Current status of end-stage renal disease care in India and Pakistan. Kidney Int Suppl 2013;3:157-60.

2 Hill NR, Fatoba ST, Oke JL, et al. Global Prevalence of Chronic Kidney Disease - A Systematic Review and Meta-Analysis. PLoS One 2016;11:e0158765.

3 Webster AC, Nagler EV, Morton RL, et al. Chronic kidney disease. The Lancet2017;389:1238-52.

4 Levey AS, Eckardt K-U, Dorman NM, et al. Nomenclature for kidney function and disease: report of a kidney disease: improving global outcomes (KDIGO) consensus conference. Kidney Int 2020;97:1117-29.

5 Mcgee J, Pandey B, Maskey A, et al. Free dialysis in Nepal: logistical challenges explored. Hemodial Int 2018;22:283-9.

6 Hwang HS, Hong YA, Yoon HE, et al. Comparison of clinical outcome between twice-weekly and Thrice-Weekly hemodialysis in patients with residual kidney function. Medicine 2016;95:e2767.

7 Sinclair A, Cimon K, Loncar M, et al. CADTH optimal use reports. dialysis modalities for the treatment of end-stage kidney disease: a health technology assessment. Ottawa (on): Canadian agency for drugs and technologies in health, 2017.

8 Wan EYF, Chen JY, Choi EPH, et al. Patterns of health-related quality of life and associated factors in Chinese patients undergoing haemodialysis. Health Qual Life Outcomes 2015;13:108.

9 Meek KP, Bergeron CD, Towne SD, et al. Restricted socia engagement among adults living with chronic conditions. Int J Env ResPub He 2018;15:158.

10 Gerogianni SK, Babatsikou FP. Social aspects of chronic renal failure in patients undergoing haemodialysis. Int J Caring Sci 2014;7:740-5.

11 Li H, Jiang Y-F, Lin C-C, Hui L, Ya-fang J, Chiu-Chu L. Factors associated with self-management by people undergoing hemodialysis: a descriptive study. Int J Nurs Stud 2014;51:208-16.

12 Moskovitch JT, Mount PF, Davies MRP. Changes in symptom burden in dialysis patients assessed using a Symptom-Reporting questionnaire in clinic. J Palliat Care 2020;35:59-65.

13 Almutary $\mathrm{H}$, Bonner A, Douglas $\mathrm{C}$. Which patients with chronic kidney disease have the greatest symptom burden? A comparative study of advanced CKD stage and dialysis modality. J Ren Care 2016;42:73-82

14 Bossola M, Pepe G, Picca A, et al. Treating symptoms to improve the quality of life in patients on chronic hemodialysis. Int Urol Nephrol 2019;51:885-7.

15 Almutary H, Bonner A, Douglas C. Symptom burden in chronic kidney disease: a review of recent literature. J Ren Care 2013;39:140-50.

16 Dąbrowska-Bender M, Dykowska G, Żuk W, et al. The impact on quality of life of dialysis patients with renal insufficiency. Patient Prefer Adherence 2018;12:577-83.

17 Hornsby BWY, Naylor G, Bess FH. A taxonomy of fatigue concepts and their relation to hearing loss. Ear Hear 2016;37 Suppl 1:136S-44.

18 Bossola M, Vulpio C, Tazza L. Fatigue in chronic dialysis patients. Semin Dial 2011;24:550-5.

19 Zalai D, Bohra M. Fatigue in chronic kidney disease: definition, assessment and treatment. Cannt J 2016;26:39-44.

20 Kraus MA, Fluck RJ, Weinhandl ED, et al. Intensive hemodialysis and health-related quality of life. Am J Kidney Dis 2016;68:S33-42.

21 Bossola M, Di Stasio E, Giungi S, et al. Fatigue is associated with serum interleukin-6 levels and symptoms of depression in patients on chronic hemodialysis. J Pain Symptom Manage 2015;49:578-85.

22 Artom M, Moss-Morris R, Caskey F, et al. Fatigue in advanced kidney disease. Kidney Int 2014;86:497-505.

23 Bonner A, Wellard S, Caltabiano M. The impact of fatigue on daily activity in people with chronic kidney disease. J Clin Nurs 2010;19:3006-15.

24 Bonner A, Wellard S, Caltabiano M. Levels of fatigue in people with ESRD living in far North Queensland. J Clin Nurs 2008;17:90-8. 
25 Horigan AE. Fatigue in hemodialysis patients: a review of current knowledge. J Pain Symptom Manage 2012;44:715-24.

26 Picariello F, Moss-Morris R, Macdougall IC, et al. The role of psychological factors in fatigue among end-stage kidney disease patients: a critical review. Clin Kidney J 2017;10:79-88.

27 Horigan AE, Schneider SM, Docherty S, et al. The experience and self-management of fatigue in patients on hemodialysis. Nephrol Nurs J 2013;40:113-22.

28 Johansen KL, Finkelstein FO, Revicki DA, et al. Systematic review of the impact of erythropoiesis-stimulating agents on fatigue in dialysis patients. Nephrol Dial Transplant 2012;27:2418-25.

29 Jhamb M, McNulty ML, Ingalsbe G, et al. Knowledge, barriers and facilitators of exercise in dialysis patients: a qualitative study of patients, staff and nephrologists. BMC Nephrol 2016;17:192.

30 Bennett S, Pigott A, Beller EM, et al. Educational interventions for the management of cancer-related fatigue in adults. Cochrane Database Syst Rev 2016;11:CD008144.

31 Kao Yu-Hsiu, Huang Yi-Ching, Chen Pei-Ying, Kao Y-H, Huang Y-C, Chen $\mathrm{P}-\mathrm{Y}$, et al. The effects of exercise education intervention on the exercise behaviour, depression, and fatigue status of chronic kidney disease patients. Health Educ 2012;112:472-84.

32 Blikman LJ, Huisstede BM, Kooijmans H, et al. Effectiveness of energy conservation treatment in reducing fatigue in multiple sclerosis: a systematic review and meta-analysis. Arch Phys Med Rehabil 2013;94:1360-76.

33 Sadeghi E, Gozali N, Moghaddam Tabrizi F, Tabrizi FM. Effects of energy conservation strategies on cancer related fatigue and health promotion lifestyle in breast CancerSurvivors: a randomized contro trial. Asian Pac J Cancer Prev 2016;17:4783-90.

34 Khozimeh FS, Navidian A, Sasanpour P, et al. The effect of training on energy conservation strategies, fatigue, and Self-Caring among women with breast cancer undergoing chemotherapy in Zahedan medical sciences hospitals in 2018-2019. J Evol Med Dent Sci 2019;8:3661-6.

35 Finlayson M, Preissner K, Cho C, et al. Randomized trial of a teleconference-delivered fatigue management program for people with multiple sclerosis. Mult Scler 2011;17:1130-40.

36 Mathiowetz VG, Finlayson ML, Matuska KM, et al. Randomized controlled trial of an energy conservation course for persons with multiple sclerosis. Mult Scler 2005;11:592-601.

37 Sharma S, Green T, Alexander KE, et al. Educational or behavioural interventions for symptoms and health-related quality of life in adults receiving haemodialysis: a systematic review. J Ren Care 2020;46:233-49.

38 Yoong SL, Wolfenden L, Clinton-McHarg T, et al. Exploring the pragmatic and explanatory study design on outcomes of systematic reviews of public health interventions: a case study on obesity prevention trials. J Public Health 2014;36:170-6.

39 Vadiraja HS, Rao RM, Nagarathna R, et al. Effects of yoga in managing fatigue in breast cancer patients: a randomized controlled trial. Indian J Palliat Care 2017;23:247-52.

40 Blikman LJ, van Meeteren J, Twisk JW, et al. Effectiveness of energy conservation management on fatigue and participation in multiple sclerosis: a randomized controlled trial. Mult Scler 2017;23:1527-41.

41 Packer TL, Brink N, Sauriol A. Managing fatigue : a six-week course for energy. Tucson, Ariz: Therapy Skill Builders, 1995.
42 Humphreys J, Janson S, Donesky DA. Theory of symptom management. In: New York NY, ed. Middle range theory for nursing, 3rd. US: Springer Publishing Company, 2014: 141-64.

43 Larson PJ, Carrieri-Kohlman V, Dodd MJ, et al. A model for symptom management. J Nurs Scholarsh 1994;26:272-6.

44 Hann DM, Jacobsen PB, Azzarello LM, et al. Measurement of fatigue in cancer patients: development and validation of the fatigue symptom inventory. Qual Life Res 1998;7:301-10.

45 Ju A, Unruh ML, Davison SN, et al. Patient-Reported outcome measures for fatigue in patients on hemodialysis: a systematic review. Am J Kidney Dis 2018;71:327-43.

46 Dahal A, Meheta RK. Fatigue experience and coping strategies among cancer patients receiving chemotherapy. Nepal Health Res Counc 2018;16:285-90.

47 Murphy EL, Murtagh FEM, Carey I, et al. Understanding symptoms in patients with advanced chronic kidney disease managed without dialysis: use of a short patient-completed assessment tool. Nephron Clin Pract 2009;111:c74-80.

48 C Cicely Saunders Institute. Palliative care outcome scale-IPOSRenal: Cicely Saunders Institute, 2016. Available: https://pos-pal.org/ maix/ipos-renal-in-english.php [Accessed 4 Sep 2020].

49 Cicely Saunders Institute. How to score IPOS: Cicely Saunders Institute, 2016. Available: https://pos-pal.org/maix/how-to-score.php [Accessed 4 Sep 2020].

50 Raj R, Ahuja K, Frandsen M, et al. Validation of the IPOS-renal symptom survey in advanced kidney disease: a cross-sectional study. J Pain Symptom Manage 2018;56:281-7.

51 Sousa VD, Rojjanasrirat W. Translation, adaptation and validation of instruments or scales for use in cross-cultural health care research: a clear and user-friendly guideline. J Eval Clin Pract 2011;17:268-74.

52 Law M, Baptiste S, McColl M, et al. The Canadian occupational performance measure: an outcome measure for occupational therapy. Can J Occup Ther 1990;57:82-7.

53 Carswell A, McColl MA, Baptiste S, et al. The Canadian occupationa performance measure: a research and clinical literature review. Can $J$ Occup Ther 2004;71:210-22.

54 Kafle Bhandari B, Pradhan RR, Pathak R, et al. Assessment of validity of SF 36 questionnaire using Nepali language to determine health-related quality of life in patients with chronic liver disease: a pilot study. Cureus 2018;10:e2925.

55 Brazier JE, Harper R, Jones NM, et al. Validating the SF-36 health survey questionnaire: new outcome measure for primary care. BMJ 1992;305:160-4.

56 Harris PA, Taylor R, Minor BL, et al. The REDCap Consortium: building an international community of software platform partners. $J$ Biomed Inform 2019;95:103208.

57 Schick-Makaroff K, Molzahn AE, Kalfoss M. Symptoms, coping, and quality of life of people with chronic kidney disease. Nephrol Nurs $J$ 2018;45:339-55.

58 Fukushima RLM, Menezes ALC, Inouye K. Quality of life and associated factors in patients with chronic kidney disease on hemodialysis. Acta Paulista de Enfermagem 2016;29:518-24.

59 Almutary H, Douglas C, Bonner A. Towards a symptom cluster model in chronic kidney disease: a structural equation approach. J Adv Nurs 2017;73:2450-61. 